\title{
Quantification of in vivo fluorescence decoupled from the effects of tissue optical properties using fiber-optic spectroscopy measurements
}

\author{
Anthony Kim \\ Mamta Khurana \\ University of Toronto \\ Department of Medical Biophysics \\ Toronto, Ontario, M5G 2M9, Canada
}

\author{
Yumi Moriyama \\ University Health Network \\ Ontario Cancer Institute \\ Campbell Family Institute for Cancer Research \\ 610 University Avenue \\ Toronto, Ontario, M5G 2M9, Canada
}

\author{
Brian C. Wilson \\ University of Toronto, \\ Department of Medical Biophysics, \\ Toronto, Ontario, M5G 2M9, Canada \\ and \\ University Health Network \\ Ontario Cancer Institute \\ Campbell Family Institute for Cancer Research \\ 610 University Avenue \\ Toronto, Ontario, M5G 2M9, Canada
}

\begin{abstract}
We present a method for tissue fluorescence quantification in situ using a handheld fiber optic probe that measures both the fluorescence and diffuse reflectance spectra. A simplified method to decouple the fluorescence spectrum from distorting effects of the tissue optical absorption and scattering is developed, with the objective of accurately quantifying the fluorescence in absolute units. The primary motivation is measurement of 5-aminolevulinic acid-induced protoporphyrin IX (ALA-PpIX) concentration in tissue during fluorescence-guided resection of malignant brain tumors. This technique is validated in phantoms and ex vivo mouse tissues, and tested in vivo in a rabbit brain tumor model using ALA-PpIX fluorescence contrast. @ 2010 Society of Photo-Optical Instrumentation Engineers. [DOI: 10.1117/1.3523616]
\end{abstract}

Keywords: fluorescence spectroscopy; turbid media; diffuse reflectance; cancer diagnosis; fluorescence quantification; tissue optics; protoporphyrin IX.

Paper 10170R received Mar. 30, 2010; revised manuscript received Oct. 16, 2010; accepted for publication Oct. 18, 2010; published online Dec. 23, 2010.

\section{Introduction}

Fluorescence measurements are of considerable interest for biomedical optics applications such as photosensitizer dosimetry during photodynamic therapy, ${ }^{1}$ fluorescence-guided surgery, ${ }^{2}$ detection of cancerous or dysplastic lesions, ${ }^{3}$ and uptake of exogenous contrast agents. ${ }^{4}$ The shape and intensity of the fluorescence spectrum contain valuable information on the identity and density of fluorophores in tissue. However, accurate measurements of fluorophore concentrations are confounded by the distorting effects of the optical absorption and scattering of tissue. Untangling the effects of this attenuation on the measured fluorescence spectrum is necessary for truly quantitative analysis.

Several methods have been reported for this purpose, many of which use the diffuse reflectance to correct the fluorescence signal. Thus, Müller et al. ${ }^{3}$ and Wu et al. ${ }^{5}$ developed a fluorescence photon migration model to derive a relationship with the diffuse reflectance that can be exploited to extract the quantitative fluorescence in tissue. In a different approach, Diamond et al. advanced the idea to use a single optical fiber for both source and collection. ${ }^{4}$ The concept is that detectable fluorescence events occur so close to the fiberoptic tip ${ }^{6}$ (probing depth $\sim 250 \mu \mathrm{m}$ for a $250-\mu \mathrm{m}$ fiber diameter) that absorption and scattering effects are minimal, analogous to how these effects

Address all correspondence to: Brian C. Wilson, University Health Network, Ontario Cancer Institute, Campbell Family Institute for Cancer Research, 610 University Avenue, Toronto, Ontario, M5G 2M9, Canada. Tel: 416-946-2952. Fax: 416-946-6529. E-mail: wilson@uhnres.utoronto.ca. are usually negligible for very thin tissue sections, as in microscopy. Empirical methods with similar underlying concepts have also been developed. Thus, Finlay et al. used the singlefiber method, together with an empirically derived correction factor that depends on the tissue optical properties at the fluorescence emission wavelength, to compensate for high tissue attenuation in the prostate during photodynamic therapy (PDT) studies. ${ }^{1}$ Weersink et al. used a fluorescence/reflectance ratio to quantify fluorophore concentration, but with the fluorescence and reflectance measured at different source-collector fiber optic distances that were optimally selected using Monte Carlo simulations. ${ }^{7}$ Ex vivo extraction techniques have also been developed that are based on solubilizing the tissue and diluting the resulting solution to the point that optical attenuation effects can be neglected. ${ }^{8}$ These ex vivo procedures are typically time-consuming and, depending on the fluorophore, may be error-prone due to tissue handling and/or cryofreezing for later processing.

In this paper, we present a noninvasive in vivo method using a simple closed-form relationship to extract the quantitative fluorescence spectrum, i.e., the fluorescence emission spectrum corrected for the optical attenuation of the tissue, and consequently to determine fluorophore concentration. The tissue optical properties, diffuse reflectance spectrum, and measured fluorescence spectrum (i.e., the raw, uncorrected fluorescence measurements that are distorted by the optical attenuation) are inputs

$1083-3668 / 2010 / 15(6) / 067006 / 12 / \$ 25.00$ C 2010 SPIE 
to the model. Since the tissue optical absorption and scattering properties must be known, a method to extract these is reviewed, using a recently reported technique. ${ }^{9}$

Our specific interest here is to develop a technique to quantitatively measure the fluorescence in situ during surgical resection of brain tumor using 5-aminolevulinic acid (ALA)induced protoporphyrin IX (PpIX). ALA, which promotes overproduction of the endogenous fluorophore PpIX in cells, has been shown to have strong selectivity to high-grade glioma cells compared to normal brain tissue. Intraoperative fluorescence imaging, mediated by ALA-PpIX, has resulted in significantly increased completeness of tumor resection, and consequently increased progression-free survival, in high-grade glioma patients. ${ }^{2}$ However, currently resection decisions are based on purely subjective and qualitative assessment of the fluorescence images. ${ }^{2,10}$ Moreover, the differences between observed "strong" and "weak" fluorescence intensities are as likely to be due to differences in tissue absorption and scattering as on varying fluorophore concentration: most such studies use short-wavelength (UV to blue) light excitation to get the brightest PpIX intensity but this region also has very high tissue absorption due to hemoglobin, ${ }^{10-12}$ and hence marked variations in light attenuation. The observed fluorescence intensity is also highly dependent on the viewing distance and angle relative to the tissue surface, especially in resection cavities where there are large surface contour variations. Hence, there is a clear need to devise more quantitative methods to evaluate the fluorescence signals to improve decision making during fluorescence-guided surgical resection to make it an objective, reproducible, and more operator-independent technique.

Much of the published work on quantifying fluorescence in tissues involves excitation wavelengths where the tissue attenuation is low $\left(\mu_{a}<1 \mathrm{~cm}^{-1}\right)$, such as in the work of Diamond et al. ${ }^{4}$ and Weersink et al. ${ }^{7}$ For our primary application of interest, namely, fluorescence-guided resection of residual brain tumor using ALA-PpIX contrast, PpIX has a maximum absorption at $400 \mathrm{~nm}$, in the region of high tissue absorption due to hemoglobin. Hence, the challenge here is to decouple the quantitative fluorescence from the tissue optical properties under high-attenuation conditions $\left(\mu_{a}>10 \mathrm{~cm}^{-1}\right.$, typical of tissues in the UV/blue wavelength range). In addition, absolute quantitation of fluorophore concentration (in this case, PpIX) is desired: few studies have attempted to retrieve absolute data in vivo from fluorescence spectra that are corrected for tissue optical properties distortion. In addition, for surgical applications, the measurements must be performed very rapidly $(<1 \mathrm{~s})$, the equipment must be portable and usable in the operating room environment, and the computation of the quantitative fluorescence must be robust and provide absolute fluorophore concentration.

To satisfy these requirements, we have derived a simple, closed-form analytical model to extract the quantitative fluorescence spectrum with excitation wavelengths in regions of high absorption. Then, to extract the true fluorescence signal, the tissue optical properties must be known at the excitation wavelength. For this, we use the technique of spectrally constrained diffuse reflectance. Fluorophore concentrations can then be calculated from the quantitative fluorescence spectrum through spectral decomposition using a priori basis spectra. The fluorescence model is implemented into a handheld fiber optic probe for investigations of ALA-PpIX tumor contrast in guided resection surgery of brain tumors. However, in principle the approach is generic to any visible/near-IR emitting fluorophores and many tissue types.

\section{Theory}

\subsection{Fluorescence Model}

The fiber optic geometry for fluorescence collection is shown in Fig. 1(a). The following fluorescence model is based on the assumption that the optical absorption at the excitation wavelength $\lambda_{x}$ is high relative to that at emission wavelength* $\lambda_{m}$. This is generally true in tissue if the excitation wavelength is in the UV to the blue end of the visible spectrum ( 380 to $450 \mathrm{~nm}$ ). As a result, most fluorophore absorption events occur close to the source fiber. The migration paths of the fluorescence emission photons at $\lambda_{m}$ are then approximated by those of the reflectance photons at $\lambda_{m}$, emitted and collected using the same fiber optic geometry. Given constant optical properties at $\lambda_{x}$ and constant fluorophore concentration, it follows that the measured fluorescence $F_{x, m}$ is linearly proportional to the diffuse reflectance $R_{m}$ at the emission wavelength:

$$
F_{x, m}=S R_{m},
$$

where $S$ is the fraction of excitation photons launched into the tissue that are absorbed and produce fluorescence.

The term $S$ can be modeled as the fraction $S_{1}$ of the total excitation photons that are retained within the tissue at steady state (i.e., those photons that are not diffusely re-emitted through the tissue surface, represented by $R_{t, x}$ ), multiplied by the fraction $S_{2}$ of the total absorbed photons that are reemitted as fluorescence. The total diffuse reflectance $R_{t, x}$ depends on the internal reflection parameter $\kappa=\left(1+r_{\mathrm{id}}\right) /\left(1-r_{\mathrm{id}}\right)$ that arises from refractive index mismatch between the tissue and the external medium, and the reduced albedo at $\lambda_{x}, a_{x}^{\prime}=\mu_{s, x}^{\prime} /\left(\mu_{a, x}+\mu_{s, x}^{\prime}\right)$. It is given by diffusion theory ${ }^{13}$ as

$$
R_{t, x}=\frac{a_{x}^{\prime}}{1+2 \kappa\left(1-a_{x}^{\prime}\right)+[1+(2 \kappa / 3)] \sqrt{3\left(1-a_{x}^{\prime}\right)}}
$$

An empirical formulation of $r_{\text {id }}$ for index-mismatched boundaries has been widely used, ${ }^{14} r_{\mathrm{id}}=-1.44 n_{\text {rel }}^{-2}+0.71 n_{\text {rel }}^{-1}$ $+0.67+0.0636 n_{\text {rel }}$, where $n_{\text {rel }}=n_{\text {tissue }} / n_{\text {external }}$. For matching internal and external refractive indices $\kappa=1$, and this was used here, since the ink-blackened epoxy surrounding the probe fibers acts as the external medium. The probe-tissue interface was assumed to be index-matched, based on published values for the refractive index of epoxy (EPO-TEK 301 epoxy: www.epotek.com) and glass fiber (FG200LCC fibers: www.thorlabs.com) used in the probe construction being in the range 1.4 to 1.5 . Note that $S_{1}$ is the fraction of photons that are not diffusely reflected out of the tissue, so that $S_{1}=\left(1-R_{t, x}\right)$.

The quantitative fluorescence $f_{x, m}$ in units of $\mathrm{nm}^{-1} \mathrm{~cm}^{-1}$ is defined here as the product of the wavelength-dependent fluorescence quantum yield $Q_{x, m}$ (the fluorescence quantum yield $Q$

\footnotetext{
*Variables at the excitation and emission wavelengths are denoted by subscripts $x$ and $m$, respectively.
} 
(a)

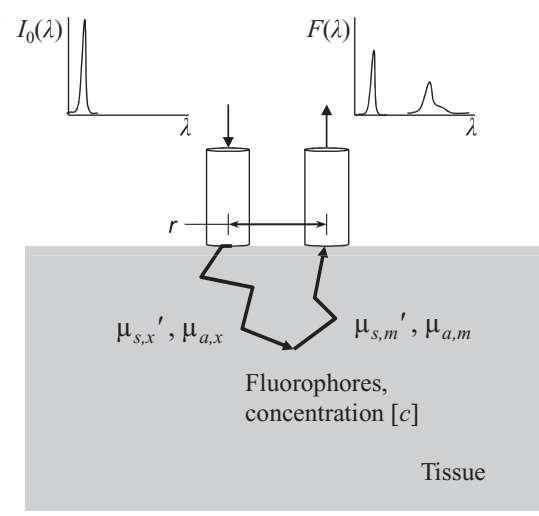

(b)

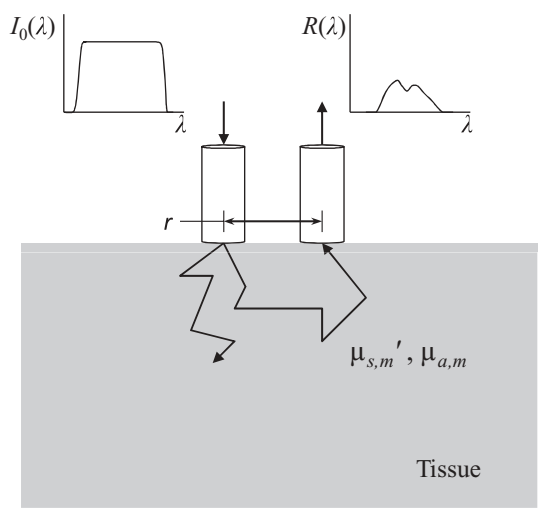

Fig. 1 Fiber optic geometry (a) for fluorescence collection and (b) for measuring the diffuse reflectance spectrum.

is related to $Q_{x, m}$ such that $Q=\int_{\lambda_{r}} Q_{x, m} d \lambda_{m}$, in other words, $Q_{x, m}$ gives the spectral shape of the fluorescence emission with the area under the curve within the wavelength range $\lambda_{r}$ of the fluorescence emission being the quantum yield) and the fluorescence absorption coefficient at the excitation wavelength $\mu_{a f, x}$. It is, therefore, an intrinsic property of the tissue fluorophores, rather than depending on the collection geometry or tissue optical properties. The fraction of total absorbed photons that undergo fluorescence $S_{2}$ is simply the quantitative fluorescence divided by the total absorption. If the fluorophore absorption contribution is negligible compared to the intrinsic tissue absorption, i.e., $\mu_{a f, x} \ll \mu_{a, x}$, then the total absorption can be approximated by $\mu_{a, x}$ :

$$
S_{2}=\frac{Q_{x, m} \mu_{a f, x}}{\mu_{a, x}}
$$

The measured (uncorrected) fluorescence can now be expressed as

$$
F_{x, m}=\left(1-R_{t, x}\right)\left(\frac{Q_{x, m} \mu_{a f, x}}{\mu_{a, x}}\right) R_{m} .
$$

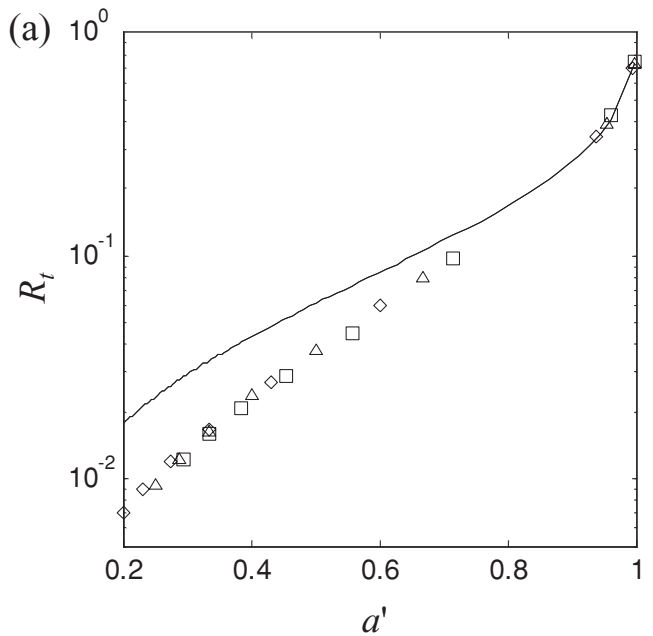

A closed-form equation for the quantitative fluorescence is then

$$
f_{x, m}=Q_{x, m} \mu_{a f, x}=\left(\frac{\mu_{a, x}}{1-R_{t, x}}\right)\left(\frac{F_{x, m}}{R_{m}}\right) .
$$

Clearly, if $\mu_{a, x} \rightarrow 0$, then $f_{x, m}$ should not go to zero. Recall, however, the underlying assumption that $\mu_{a, x}$ is high, so that Eq. (5) is invalid at low excitation absorption and the model is not applicable.

Some caution is also necessary in using the diffusion theory model in Eq. (2) for the total diffuse reflectance. For high reduced albedo, i.e., $a^{\prime}>0.5$, the relation has been shown to be accurate; however, in this paper the reduced albedo is often $<0.5$ due to the strong absorption in the excitation band. ${ }^{15}$ Despite this, Eq. (2) can still be used to accurately calculate the quantitative fluorescence. Figure 2(a) shows $R_{t}$ plotted versus $a^{\prime}$, using both diffusion theory and Monte Carlo simulations. The Monte Carlo simulations modeled a pencil light beam incident on an optically semiinfinite turbid medium, using the on-line C-code developed by Jacques. ${ }^{16}$ At low $a^{\prime}$, the diffusion theory prediction of $R_{t, x}$ deviates significantly from the Monte Carlo

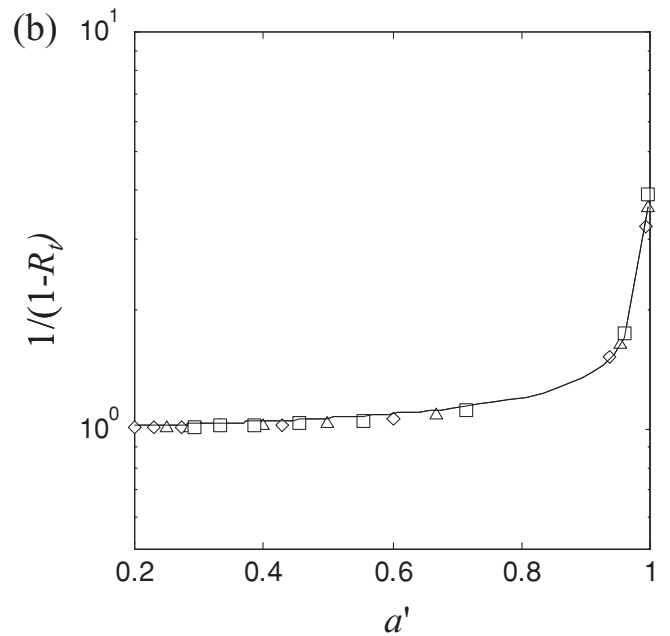

Fig. 2 (a) Diffusion theory calculations of $R_{t}$ versus $\alpha^{\prime}$ (solid line) compared with Monte Carlo simulation for $\mu_{s}^{\prime}=15,20$, and $25 \mathrm{~cm}^{-1}$ (symbols $\diamond, \Delta, \square$, respectively), while varying $\mu_{a}$ from 0.1 to $60 \mathrm{~cm}^{-1}$; and (b) diffusion theory and Monte Carlo calculations for $1 /\left(1-R_{t}\right)$. 
simulations, with an average fractional difference of $85 \%$ over the range of $a^{\prime}$ shown in Fig. 2. However, since Eq. (5) uses the term $1 /\left(1-R_{t, x}\right)$ rather than $R_{t, x}, 1 /\left(1-R_{t}\right)$ was plotted against $a^{\prime}$ [Fig. 2(b)] to determine if diffusion theory is suitable for calculating this factor. This shows that even with high absorption $\left(\mu_{a} \gg 1\right)$ and hence low albedo, diffusion theory performs well at producing accurate values for the correction factor $1 /\left(1-R_{t}\right)$, with an average fractional difference of only $2.3 \%$, justifying the use of Eq. (2) in the fluorescence model.

The corrected emission spectrum $f(\lambda)$ can be used to calculate the fluorophore concentration, $c$, given the $a$ priori fluorescence basis spectrum $b(\lambda)$ for unit concentration. The relation is

$$
\mathbf{f}=\mathbf{b} c,
$$

where $\mathbf{f}$ and $\mathbf{b}$ are $f(\lambda)$ and $b(\lambda)$ in column vector form. Solving for $c$,

$$
c=\left(\mathbf{b}^{T} \mathbf{b}\right)^{-1} \mathbf{b}^{T} \mathbf{f},
$$

where $T$ indicates the matrix transpose. Generalizing to $N$ fluorophores with distinct spectra, a basis matrix $\mathbf{B}=\left[\mathbf{b}_{1} \mathbf{b}_{2} \ldots \mathbf{b}_{N}\right]$ can be built with the individual fluorophore basis spectra as columns, with a corresponding fluorophore contribution vector $\mathbf{c}=\left[c_{1} c_{2} \ldots c_{N}\right]^{T}$. Solving for $\mathbf{c}$,

$$
\mathbf{c}=\left(\mathbf{B}^{T} \mathbf{B}\right)^{-1} \mathbf{B}^{T} \mathbf{f} .
$$

Alternatively, the vector $\mathbf{c}$ can be forced to consist of all-positive scalar values by replacing Eq. (8) with a nonnegative leastsquares algorithm.

For this work, which focuses on fluorescence guidance for the resection of brain tumor, the two main components in the fluorescence spectrum are the autofluorescence of brain tissues and the fluorescence of protoporphyrin IX. The autofluorescence spectral shape was averaged from 10 measurements made in ex vivo murine cortical brain tissue (without ALA). In the wavelength range 600 to $720 \mathrm{~nm}$, these spectra decreased approximately linearly with wavelength. The basis spectrum $\mathbf{b}_{\mathrm{PpIX}}$ for $1 \mu \mathrm{g} / \mathrm{mL}$ of PpIX (Sigma-Aldrich, Oakville, Ontario, Canada) was obtained from measurements in an Intralipid (Fresenius Kabi, Uppsala, Sweden) calibration phantom with known optical properties. Hence, for this application the basis matrix is $\mathbf{B}=\left[\begin{array}{lll}-\lambda & 1 & \mathbf{b}_{\mathrm{PpIX}}\end{array}\right]$ and the fluorophore contribution vector is $\mathbf{c}=\left[\begin{array}{lll}c_{\lambda} & c_{1} & c_{\mathrm{PpIX}}\end{array}\right]$, where $\lambda$ is the wavelength vector in the range 600 to $720 \mathrm{~nm}, 1$ is the unit vector, $c_{\lambda}$ and $c_{1}$ parameterize the autofluorescence as a linear function of wavelength, and $c_{\text {PpIX }}$ gives the $[\mathrm{PpIX}]$ in $\mu \mathrm{g} / \mathrm{mL}$. The $\mathbf{c}$ vector is forced to be positive using a nonnegative least-squares algorithm. The autofluorescence spectrum was included in the model primarily to subtract the background in determining [PpIX]. For applications where the autofluorescence itself is of interest, a more sophisticated model may be required, such as a linear combination of the spectral shapes of endogenous fluorophores that are expected to be in the target tissue.

To recapitulate, the assumptions in the fluorescence model are that

1 Diffusely reflected and fluorescence photons traverse similar path lengths for the same fiberoptic separations, given that $\mu_{a, x} \gg \mu_{a, m}$, which is generally true in tissue if the excitation wavelength is in the UV to blue range.
2 The value of $\mu_{a, x} \gg \mu_{a f, x}$. In many cases, the fluorophore contribution to $\mu_{a, x}$ may be small compared to the high absorption of hemoglobin in the range 380 to $450 \mathrm{~nm}$, but this should be considered based on the expected maximum concentration of the fluorophore of interest. In the situation where fluorophore absorption at $\lambda_{x}$ is significant compared with tissue absorption, the albedo becomes $a_{x}^{\prime}=\mu_{s, x}^{\prime} /\left(\mu_{a, x}+\mu_{a f, x}+\mu_{s, x}^{\prime}\right)$, and Eq. (3) becomes $S_{2}=\left(Q_{x, m} \mu_{a f, x}\right) /\left(\mu_{a, x}+\mu_{a f, x}\right)$, and a priori knowledge of the quantum yield is required to solve for the quantitative fluorescence in Eq. (4).

\subsection{Extraction of Tissue Optical Properties Using Spectrally constrained Diffuse Reflectance}

The fluorescence model of Eq. (5) requires spectral values for the excitation tissue optical properties $\mu_{a, x}$ and $\mu_{s, x}^{\prime}$. We recently reported a novel method to measure spectrally resolved tissue optical absorption and reduced scattering coefficients in the visible and near-IR range employing a series of fiber optic sourcecollector pairs to measure the steady state diffuse reflectance spectrum. The source fiber delivers broadband (white) light in the spectral range of interest and the diffuse reflectance spectrum is detected by the collector fiber located at a radial distance $r$ from the source, measured using a spectrometer. Since there is only one reflectance measurement per wavelength, solving for $\mu_{a}(\lambda)$ and $\mu_{s}^{\prime}(\lambda)$ relies on applying a spectral constraint, i.e., using a priori knowledge of the shapes of the absorption and reduced scattering coefficient spectra in the forward model. The approach here is to determine $\mu_{a}(\lambda)$ and $\mu_{s}^{\prime}(\lambda)$ over a spectral range (450 to $720 \mathrm{~nm}$ ) to provide a good model fit, and then to extract $\mu_{a}\left(\lambda_{x}\right)$ and $\mu_{s}^{\prime}\left(\lambda_{x}\right)$ by extrapolating to the excitation wavelength (here, $\lambda_{x}=405 \mathrm{~nm}$ ). Using this approach to find the tissue optical properties requires that the absorption from the fluorophore(s) do not significantly distort the tissue diffuse reflectance spectrum. The method described in the following was previously published, ${ }^{9,15}$ so that only the salient points are outlined here.

The absorption spectrum can be modeled as a linear combination of the separate chromophore contributions. Here, it is expressed using the hemoglobin concentration and an oxygen saturation term:

$\mu_{a}(\lambda)=c_{\mathrm{Hb}}\left[\mathrm{StO}_{2} \mu_{a}^{\mathrm{oxyHb}}(\lambda)+\left(1-\mathrm{StO}_{2}\right) \mu_{a}^{\mathrm{deoxyHb}}(\lambda)\right]$,

where $\mu_{a}^{\mathrm{oxyHb}}(\lambda)$ and $\mu_{a}^{\mathrm{deoxyHb}}(\lambda)$ are the wavelengthdependent absorption coefficients of oxyhemoglobin and deoxyhemoglobin, respectively, at concentrations of $1 \mathrm{~g} / \mathrm{L} ; c_{\mathrm{Hb}}$ is the total hemoglobin concentration; and $\mathrm{StO}_{2}$ is the oxygenation fraction. Water absorption is considered negligible in the range 450 to $720 \mathrm{~nm}$.

The reduced scattering coefficient spectrum from bulk tissue has been shown to fit well to a simple wavelength-dependent power law, given by

$$
\mu_{s}^{\prime}(\lambda)=A \lambda^{-k}
$$

where $A$ and $k$ are constants. The $a$ priori absorption and reduced scattering spectra are then combined in a forward model of 
the diffuse reflectance, and a Levenberg-Marquardt algorithm is applied to extract the free parameters. A simple approach to develop a forward model is to use the diffusion theory equation for spatially resolved, steady state diffuse reflectance $R$. Here, the radial distance $r$ is fixed and the optical properties $\mu_{a}(\lambda)$ and $\mu_{s}^{\prime}(\lambda)$ vary according to wavelength:

$$
\begin{aligned}
R(\lambda)= & \frac{a^{\prime}}{4 \pi}\left[z_{0}\left(\mu_{\mathrm{eff}}+\frac{1}{\rho_{1}}\right) \frac{\exp \left(-\mu_{\mathrm{eff}} \rho_{1}\right)}{\rho_{1}^{2}}\right. \\
& \left.+\left(z_{0}+2 z_{b}\right)\left(\mu_{\mathrm{eff}}+\frac{1}{\rho_{2}}\right) \frac{\exp \left(-\mu_{\mathrm{eff}} \rho_{2}\right)}{\rho_{2}^{2}}\right],
\end{aligned}
$$

where $z_{0}=1 / \mu_{s}^{\prime}, \mu_{\text {eff }}(\lambda)=\left[3 \mu_{a}(\lambda) \mu_{s}^{\prime}(\lambda)\right]^{1 / 2}, \mu_{a}(\lambda)$ and $\mu_{s}^{\prime}(\lambda)$ are given by Eqs. (9) and (10), $\rho_{1}^{2}=z_{0}^{2}+r^{2}$, and $\rho_{2}^{2}$ $=\left(z_{0}+2 z_{b}\right)^{2}+r^{2}$. The parameters $z_{0}, \rho_{1}, \rho_{2}, z_{b}$, and $\mu_{\text {eff }}$ are all wavelength dependent. The extrapolated boundary distance is given by $z_{b}=2 \kappa D$, where $D$ is the diffusion constant given by $D=\left(3 \mu_{s}^{\prime}\right)^{-1}$. This version of the diffusion constant was selected for reasons given in our previous study on the measurement of tissue optical properties. ${ }^{9}$

The free parameters are, therefore, the hemoglobin concentration, oxygen saturation, and scattering parameters. This is not quite as simple as applying the inverse algorithm to any $r$, since there is a restricted range of validity for each $r$ that is set by the peak of the reflectance versus $\mu_{s}^{\prime}$ curve, and the diffusion theory model breakdown at low $\mu_{s}^{\prime}$. However, the dynamic range of the measurement can be increased by using reflectance spectra measured at several different $r$ values with overlapping ranges of validity. We previously reported ${ }^{9}$ successful application of this approach, using $r$ values of 260,520 , and $780 \mu \mathrm{m}$ to span large dynamic ranges for $\mu_{a}$ and $\mu_{s}^{\prime}$. For these studies, we selected $r=260$ and $520 \mu \mathrm{m}$, for which the ranges of validity are approximately $\mu_{s}^{\prime}=10$ to $50 \mathrm{~cm}^{-1}$ and $\mu_{a}=0$ to $10 \mathrm{~cm}^{-1}$, which span the range of interest for measurements ${ }^{15}$ in brain for wavelengths from 450 to $720 \mathrm{~nm}$.

In previous work, we calculated the effective penetration depth $\delta$ (the depth traversed by $90 \%$ of the detected photons) for this probe geometry by Monte Carlo simulation in diffuse reflectance mode. This depended on the optical properties and ranged between 0.46 and $1.64 \mathrm{~mm}$ over the tested ranges ( $\mu_{a}=0.1$ to $10 \mathrm{~cm}^{-1}$ and $\mu_{s}^{\prime}=5$ to $20 \mathrm{~cm}^{-1}$ ).

\section{Materials and Methods}

\subsection{Measurement System and Calibration/Performance Tests}

The instrument was designed for clinical use to quantify fluorescence signal during resection surgery of malignant glioma using ALA-induced PpIX as the tumor-targeting fluorophore. A photograph of the probe is shown in Fig. 3(a), with a schematic of the tip geometry in Fig. 3(b). A linear array of four optical fibers (FG200LCC: ThorLabs, Newton, New Jersey), each spaced $260 \mu \mathrm{m}$ apart, was fixed by epoxy into 18-gauge stainless steel tubing (outer diameter $=1.1 \mathrm{~mm}$, inner diameter $=1.06 \mathrm{~mm}$ ). The silica core of each fiber is $200 \mu \mathrm{m}$, and the numerical aperture is 0.22 . The tubing was affixed to a stainless steel handle, with the four fibers extending $3 \mathrm{~m}$ to SMA905 connectors.

The control system [Fig. 3(c)] directs the flow of optical signals into and out of the probe. The white-light source for the diffuse reflectance measurements and the 405-nm source for fluorescence excitation are LEDs (LedEngin Inc., Santa Clara, California), controlled by computer via an analog data output card (Measurement Computing, Norton, Massachusetts). The fluorescence LED was filtered with a 550-nm short-pass filter (Edmund Optics, Barrington, New Jersey). The spectrometer was a USB2000 + (Ocean Optics, Dunedin, Florida). The control system unit consisted of these elements in a custom-built aluminum enclosure. The completed instrument met electrical safety requirements of the Canadian Standards Association.

A custom Labview (National Instruments, Austin, Texas) application on a laptop computer was used to control the LED signals and spectrometer acquisition. The program acquired the following sequence of measurements:

1 diffuse reflectance spectrum at $r=260 \mu \mathrm{m}$

2 diffuse reflectance spectrum at $r=520 \mu \mathrm{m}$

3 fluorescence spectrum (405-nm excitation) at $r$ $=260 \mu \mathrm{m}$

4 background signal from ambient light

A measurement sequence takes $\sim 0.6 \mathrm{~s}$. Here, the white-light reflectance and fluorescence spectra at $r=260 \mu \mathrm{m}$ were used for the quantitative fluorescence and spectral fitting: Eqs. (5) and (7). The reflectance spectra at $r=260$ and $520 \mu \mathrm{m}$ were used to determine the tissue optical properties using the spectrally constrained diffuse reflectance method: Eqs. (9) to (11).

The reflectance measurements were calibrated according to a published method using fractionated Intralipid samples, such that the absolute reflectance is determined ${ }^{9}\left[\mathrm{~cm}^{-2}\right]$. The fluorescence measurements were also calibrated using an Intralipid liquid phantom with known $\mu_{a, x}, \mu_{s, x}^{\prime}$, and [PpIX]. The published ${ }^{17}$ fluorescence quantum yield of $0.5 \%$ for PpIX was used to scale the quantitative fluorescence measurements to units of $\mathrm{nm}^{-1} \mathrm{~cm}^{-1}$.

Repeatability performance tests were carried out as follows. Silicone-based solid phantoms with titanium dioxide $\left(\mathrm{TiO}_{2}\right)$ for optical scattering were formulated according to a protocol developed at the University of California, Irvine (www.bil.uci.edu/ntroi/pubs/pdf/si_recipe.pdf). The probe tip was placed in contact with the silicone- $\mathrm{TiO}_{2}$ phantom and a measurement taken. The entire system, including the computer, was then shut down, and the probe leads were disconnected and then reattached. The system was rebooted and the phantom measurements repeated. This was done five times. The standard deviations of the phantom measurements of the diffuse reflectance (at $r=260$ and $520 \mu \mathrm{m}$ ), normalized to the mean values were 2.1 and $2.0 \%$, respectively. The standard deviation of the reemitted fluorescence excitation light as measured from the silicone phantom was $1.2 \%$. These tests were done by holding the probe approximately perpendicular to the silicon phantom surface by hand: there was negligible dependence on the pressure applied or on the different positions on the phantom.

\subsection{Phantom Experiments to Test the Accuracy of the Fluorescence Model}

Phantom experiments were carried out to test the fluorescence model. Intralipid provided the tissue-like background scattering, 

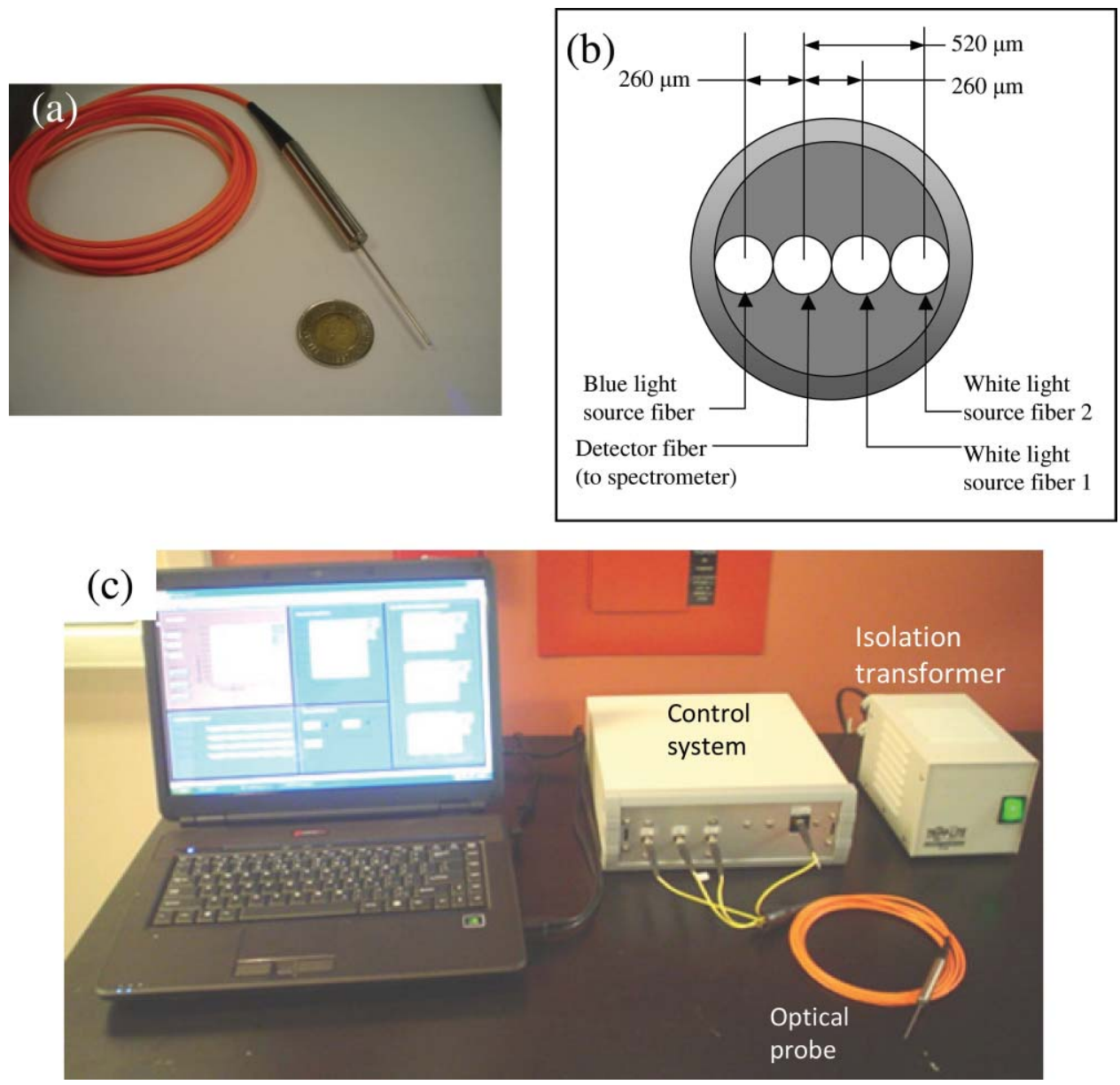

Fig. 3 (a) Photograph of the handheld probe, (b) schematic of the probe tip geometry, and (c) photograph of the probe and complete instrument.

yellow food coloring (McCormick Canada, London, Ontario) was used to vary the absorption and protoporphyrin IX was used as the target fluorophore.

The absorption coefficients of the yellow dye were measured using a spectrophotometer (Cary 5000, Varian Inc., Palo Alto, California). The reduced scattering coefficient spectrum of Intralipid was quantified previously as a function of concentration. ${ }^{9}$ A set of nine phantoms were prepared, yielding the optical properties shown in Table 1.

We mixed PpIX in six concentrations $(5,2.5,1.25,0.625$, 0.3125 , and $0.15625 \mu \mathrm{g} / \mathrm{mL}$ ) for each set of nine optical properties, for a total of 54 separate phantoms. Probe measurements were taken in each of the 54 phantoms and then Eq. (5) was applied to extract the quantitative fluorescence spectra and PpIX concentrations. Measurements were taken in order of increasing [PpIX] (in principle, the order of measurement should have no bearing on the results, since the instrument is very stable over time), with the probe tip cleaned with distilled water between measurements. Images of the phantom surfaces (at $[\mathrm{PpIX}]=5 \mu \mathrm{g} / \mathrm{mL}$ ) were also taken using a fluorescence stereomicroscope (MZ FLIII: Leica, Wetzlar, Germany) to compare the observed fluorescence intensities with the quantitative measurements using the fiber optic probe technique.

\subsection{Ex Vivo Tissue Validation Studies}

All animal experiments were carried out with institutional animal-care approvals (University Health Network, Toronto). A mouse tumor model was used to validate the accuracy of the probe and model in measuring photosensitizer concentration in various tissue types, again with PpIX as the target fluorophore, and compared with measurements of diluted, solubilized tissue in a cuvette-based fluorometer using an established protocol. ${ }^{8}$

\subsubsection{Tumor induction}

Five male mice $(20 \mathrm{~g})$ were anesthetized with $2 \%$ isoflurane and placed on a warming blanket. The skin at the injection site was swabbed with $70 \%$ ethanol, and $10^{6}$ B16 melanoma cells in $20 \mu \mathrm{L}$ of phosphate-buffered saline (PBS) were injected subcutaneously into the left flank.

\subsubsection{PplX measurement in different organs}

After tumors had grown for 7 days to approximately 4 to $7 \mathrm{~mm}$ diameter, each mouse was injected via the tail vein with $100 \mathrm{mg} / \mathrm{kg}$ ALA (Sigma-Aldrich) at $0.5,1,2,3$, or $4 \mathrm{~h}$ before sacrifice, which ensured a large range of PpIX concentration across all tissue samples. The mice were sacrificed by cervical 
Table 1 Phantom optical properties at $\lambda_{x}=405 \mathrm{~nm}$ and $\lambda_{m}=635 \mathrm{~nm}$ prior to the addition of PpIX; the addition of PpIX at the maximum concentration increases the absorption coefficient by $<2 \%$.

\begin{tabular}{lcccc}
\hline & $\mu_{a, x}\left(\mathrm{~cm}^{-1}\right)$ & $\mu_{s, x}^{\prime}\left(\mathrm{cm}^{-1}\right)$ & $\mu_{a, m}\left(\mathrm{~cm}^{-1}\right)$ & $\mu_{s, m}^{\prime}\left(\mathrm{cm}^{-1}\right)$ \\
\hline Phantom A & 20 & 15 & 0.02 & 8.7 \\
Phantom B & 20 & 20 & 0.04 & 11.6 \\
Phantom C & 20 & 25 & 0.06 & 14.5 \\
Phantom D & 40 & 15 & 0.02 & 8.7 \\
Phantom E & 40 & 20 & 0.04 & 11.6 \\
Phantom F & 40 & 25 & 0.06 & 14.5 \\
Phantom G & 60 & 15 & 0.02 & 8.7 \\
Phantom H & 60 & 20 & 0.04 & 11.6 \\
Phantom I & 60 & 25 & 0.06 & 14.5 \\
\hline
\end{tabular}

dislocation under isoflurane general anesthesia. Blocks $(\sim 5$ to $10 \mathrm{~mm}$ across) of each tissue (brain, heart, kidney, liver, muscle, skin, and tumor) were rapidly excised under subdued lighting and probe measurements were taken on each sample at three different positions. The samples were weighed, placed into cryotubes, snap frozen in liquid nitrogen, and stored at $-70^{\circ} \mathrm{C}$ in a light-tight container until the tissue solubilization procedure was carried out in batches.

\subsubsection{Tissue solubilization}

The tissue solubilization protocol for measurement of absolute fluorophore concentration was developed by Lilge et al. ${ }^{8}$ Each tissue sample was combined with $2 \mathrm{~mL}$ Solvable (Perkin Elmer, Waltham, Massachusetts) and placed in an undulating water bath at $50^{\circ} \mathrm{C}$ for $1 \mathrm{~h}$. The resulting material was homogenized (Tissue Tearor, Biospec Products, Bartlesville, Oklahoma) in the original vial. We combined $200 \mu \mathrm{L}$ of this homogenate with $3 \mathrm{~mL}$ of distilled water and $1 \mathrm{~mL}$ of Solvable. This solution was incubated in the water bath at $50^{\circ} \mathrm{C}$ for $1 \mathrm{~h}$. The optical density was measured and diluted to $<0.1$ if required. The resulting solution was transferred to a quartz cuvette and placed in a fluorometer (Fluorolog: Jobin Yvon, Edison, New Jersey), using an excitation wavelength of $401 \mathrm{~nm}$. A look-up curve was constructed by measuring known concentrations of PpIX in $75 \% / 25 \%$ distilled water/Solvable solution, with the detector nonlinearity taken into account. The estimated accuracy of the solubilization technique for measuring $[\mathrm{PpIX}]$ is $\pm 13.5 \%$.

\subsection{In Vivo Brain Tumor Model}

An intracranial brain tumor model (VX2) in rabbits was used to test the instrument sensitivity and to get baseline measurements in the in vivo surgical setting for the intended application of fluorescence-guided resection of malignant glioma. Female New Zealand white rabbits $\sim 4 \mathrm{~kg}$ (Charles River, Montreal, Quebec, Canada) were used.

\subsubsection{Tumor induction}

The VX2 tumor cells were propagated in the flank muscle and harvested at $\sim 1 \mathrm{~cm}$ diameter. VX2 cells were extracted with a strainer and implanted into the brain within $2 \mathrm{~h}$. For this, the antibiotic Baytril (Bayer, Toronto, Ontario, Canada) at $7.5 \mathrm{mg} / \mathrm{kg}$ was administered subcutaneously (SQ) 1 day prior and for 3 to 5 days after cell implantation. Immediately prior to induction, $10 \mathrm{mg} / \mathrm{kg}$ of the sedative acepromazine was administered SQ, and the animals were then anaesthetized and maintained with 1 to $2.5 \%$ inhaled isoflurane as required. The site was shaved and swabbed with Betadine. The head was affixed to a rabbitadapted stereotactic frame. A 2-cm midline incision was made, the scalp reflected, and the cranium exposed. Using a handheld drill with a 1-mm-diam drill bit, a burr hole was performed over the right hemisphere, anterior to the coronal suture and $5 \mathrm{~mm}$ to the right of the bregma, leaving the dura intact. A $100-\mathrm{mL}$ Hamilton syringe was introduced to a depth of $2 \mathrm{~mm}$ beneath the dura and VX2 cells in $50 \mu \mathrm{L}$ PBS were inoculated intracerebrally, over a period of 2 to 3 min under low manual pressure to avoid mechanical damage. Bone wax was used to close the burr hole and the incision was closed with sutures. After surgery the animals were monitored continually until fully recovered and buprenorphine $(0.05 \mathrm{mg} / \mathrm{kg} \mathrm{SQ})$ was administered for analgesia every $8 \mathrm{~h}$ for the first $24 \mathrm{~h}$.

\subsubsection{Craniotomy and fluorescence measurements}

At 7 to 9 days after VX2 implantation, animals underwent craniotomy surgery to expose the tumor. For this, the rabbit was injected intravenously (i.v.) with $20 \mathrm{mg} / \mathrm{kg}$ ALA (Sigma-Aldrich) as a solution in PBS buffered to $\mathrm{pH} 4$ to 5. Prior to anesthesia, acepromazine (10 mg/kg SQ) was given. For surgery, the animals were brought under general anesthesia and maintained with inhaled isoflurane. The eyes were lubricated with tear gel. The heart rate and oxygen saturation were monitored with a pulse oximeter and blood pressure monitor. The scalp was shaved and Betadine was applied to the surgical site. A 2 to $4 \mathrm{~cm}$ incision was made in the scalp along the midline. A craniotomy was performed using the handheld drill with a 1-mm drill bit. Gentle suction was used to remove irrigating fluid and bone dust. The bone flap was gently elevated, aiming to keep the entire dura intact. Once the bone flap was removed, both left and right hemispheres were exposed and the dura was cut using microsurgery scissors and forceps. Under dim room lighting, 10 probe measurements were taken near the tumor cell injection site, with an additional 10 measurements in normal brain in the contralateral hemisphere to the injection site. The animals were sacrificed postsurgery (i.v. Euthanyl, $1 \mathrm{~mL} / \mathrm{kg}$ ).

\section{Results}

\subsection{Phantom Studies}

This set of experiments was used to validate the fluorescence model of Eqs. (5) and (7), with a priori knowledge of the optical properties at the excitation wavelength. The measured fluorescence spectra $F_{x, m}\left(\lambda_{m}\right)$ for the set of nine phantoms (A to I), all with a PpIX concentration of $5 \mu \mathrm{g} / \mathrm{mL}$, is shown in Fig. 4(a). Applying Eq. (5) to the data produced the quantitative fluorescence spectra $f(\lambda)$ shown in Fig. 4(b). The root mean square 

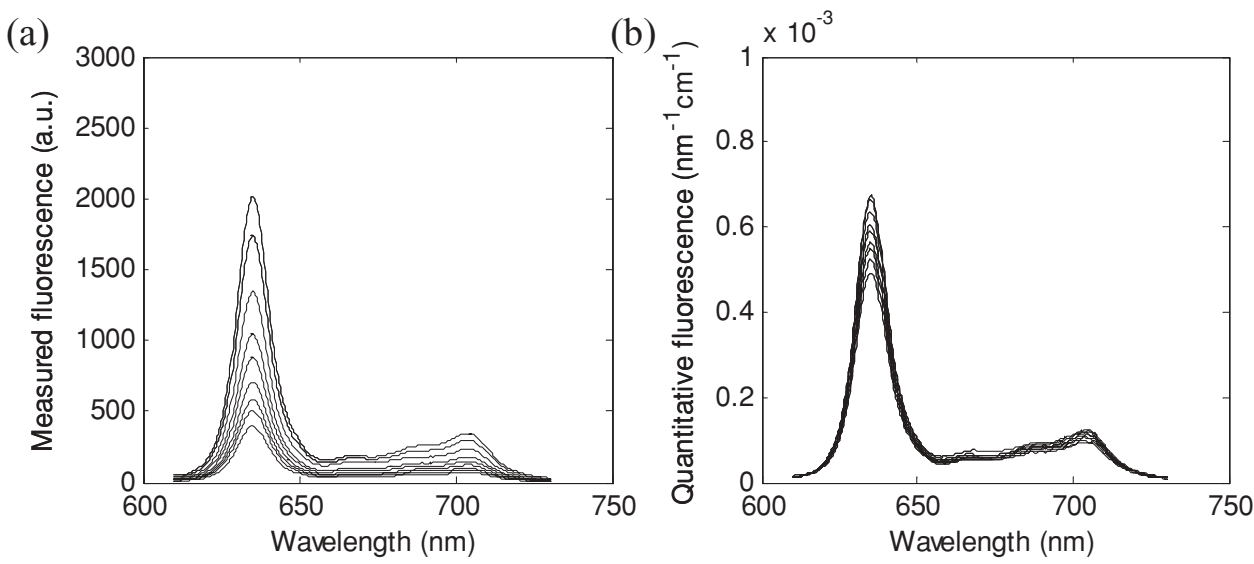

Fig. 4 (a) Measured fluorescence $F_{x, m}\left(\lambda_{m}\right)$ and (b) model-derived absolute quantitative fluorescence spectra $f(\lambda)$ for phantoms A to I at [PpIX] $=5 \mu \mathrm{g} / \mathrm{mL}$.

deviation on the mean at the $635-\mathrm{nm}$ peak was $\pm 52.3 \%$ for the measured fluorescence and $\pm 10.1 \%$ for the model-derived estimate.

Fluorescence images of the phantoms are shown in Fig. 5 at a fixed PpIX concentration $(5 \mu \mathrm{g} / \mathrm{mL})$ to give an idea of the visual appearance of the fluorescence intensity variations due to changes in the optical properties. The most marked difference, corresponding to a factor of $4: 1$ in intensity, is between phantoms $\mathrm{C}$ (highest $\mu_{s, x}^{\prime}$, lowest $\mu_{a, x}$ ) and $\mathrm{G}$ (lowest $\mu_{s, x}^{\prime}$, highest $\mu_{a, x}$ ).

The measured fluorescence intensity at the 635-nm PpIX peak from each of the 54 phantoms is shown as a function of the known PpIX concentration in Fig. 6(a), while Fig. 6(b) shows a similar graph for the PpIX concentration derived from the model applying Eqs. (5) and (7) to the measured data. Linear fits to these data show a marked increase in the goodness of fit from $R^{2}=0.639$ to 0.976 . The root mean square ( $\mathrm{rms}$ ) deviation from the mean and the maximum deviation from the mean, each normalized to the mean value, were calculated for the $5 \mu \mathrm{g} / \mathrm{mL}$ data (Table 2). This shows a five to seven fold reduction in these metrics in applying the model to the raw data.

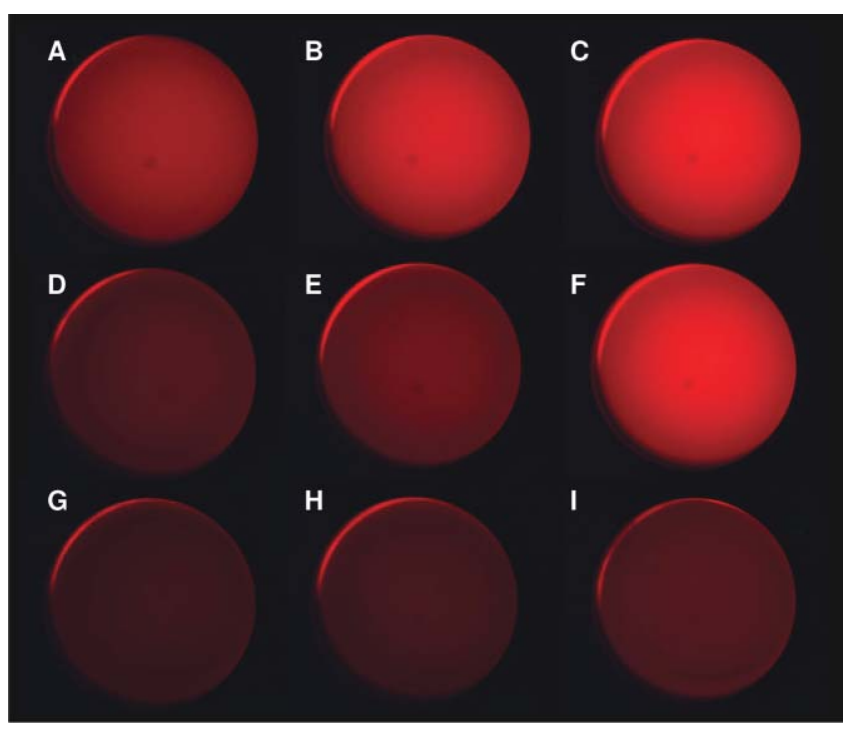

Fig. 5 Fluorescence images of phantoms A to I at $[\mathrm{PpIX}]=5 \mu \mathrm{g} / \mathrm{mL}$.

\subsection{Ex Vivo Tissue Studies}

Figure 7(a) shows the measured 635-nm PpIX peak fluorescence signal plotted against the PpIX concentration measured using the tissue solubilization technique for 34 tissue samples. ${ }^{\dagger}$ The data show large scatter, between tissues as would be expected, but also for individual tissues. The coefficient of determination was $R^{2}=0.255$ for the best line fit through the uncorrected fluorescence data in Fig. 7(a). Applying the quantitative fluorescence model gives marked improvement in the correlation. Thus, the coefficient of determination for all tissues lumped together is $R^{2}$ $=0.709$ fitting the data to the unity line, demonstrating that the technique compensates substantially, although not completely, for the variations in tissue optical properties. The best line fit through the origin, which has a slope of 0.85 , is also included in Fig. 7(b) with $R^{2}=0.871$. Note that the best line fit is not the unity line; this may be due to a difference in the fluorescence quantum yield of PpIX in tissue and in the Intralipid calibration phantom.

Note also the large range of absorption and scattering coefficients between tissues at the excitation wavelength: 7.0 to 68.3 and 5.3 to $26.3 \mathrm{~cm}^{-1}$, respectively (including data between the first and ninth deciles). It is also useful to check the optical property ranges compared to the validated dynamic range of the spectrally constrained diffuse reflectance method. The absorption spectrum as calculated from the diffuse reflectance spectrum (450 to $720 \mathrm{~nm}$ ) ranged from 0.091 to $6.64 \mathrm{~cm}^{-1}$ and the reduced scattering spectrum ranged from 4.9 to $22.9 \mathrm{~cm}^{-1}$ across all tissue measurements. Note that lower limit of the $\mu_{s}^{\prime}$ range is out of the dynamic range of the spectrally constrained diffuse reflectance method (recall that the $\mu_{s}^{\prime}$ dynamic range is from 10 to $50 \mathrm{~cm}^{-1}$ ). Both the nonunity slope and the large scatter in the [PpIX] data may be due to the tissue optical properties lying somewhat out of range of the validated dynamic range of the model. Resolving this would require ${ }^{9}$ an additional fiber separation to decrease the lower limit of the dynamic range for $\mu_{s}^{\prime}$; however, this necessitates a larger probe diameter.

\footnotetext{
${ }^{\dagger}$ There were no data for liver at $4 \mathrm{~h}$ due to a tissue handling error during the solubilization stage.
} 

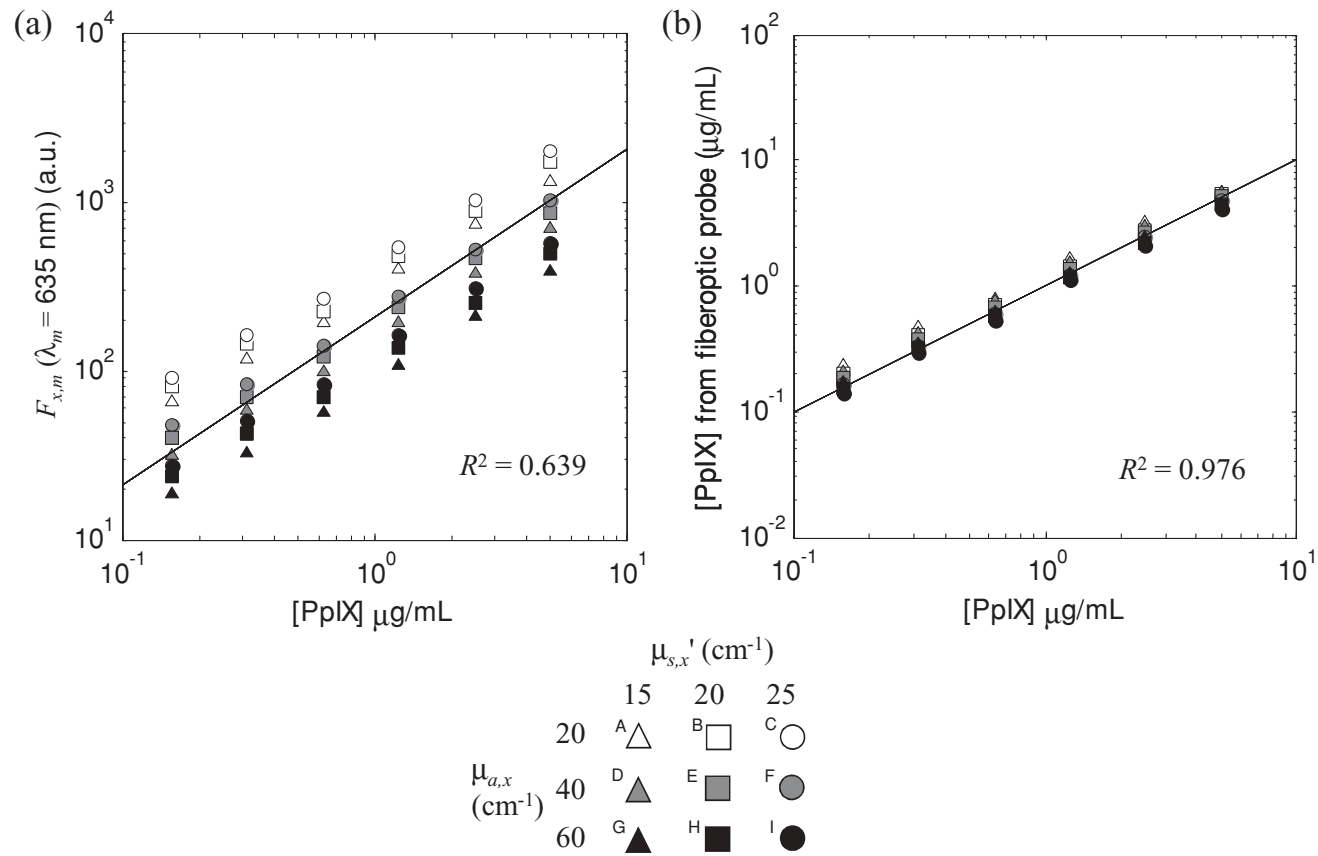

Fig. 6 (a) Measured phantom fluorescence $F_{x, m}$, at $635 \mathrm{~nm}$ and (b) estimated fluorophore concentration using the fluorescence model and spectral fitting using a basis spectrum for PpIX. The line in (a) represents the best straight-line fit through the origin; the dashed line in (b) is the unity line.

\subsection{In Vivo Studies}

Table 3 shows data in four rabbits for the maximum and minimum [PpIX] estimates around the tumor injection site and in the contralateral normal brain, making 10 different measurements in each case. Figure 8 shows representative spectra, with a strongly positive signal in the tumor site and low signal in the contralateral normal brain. The objectives were to demonstrate that the probe can detect fluorescence in vivo, in both tumor and normal tissue, as well as to obtain baseline measurements of the PpIX concentration to inform the clinical studies in glioma patients during fluorescence-image-guided surgery. The ratio of the average PpIX concentration between tumor and normal measurements was approximately $35: 1$, which is the same order of magnitude as reported in other brain tumor models using the ex vivo tissue solubilization technique with similar dose parameters. ${ }^{18}$ It is also seen that there is considerable point-to-point variation in the measured values, showing the heterogeneity of ALA uptake and/or PpIX synthesis throughout

Table 2 The rms deviation from the mean and maximum deviation from the mean, comparing the raw fluorescent image intensity, the uncorrected probe measurement at $635 \mathrm{~nm}$, and the model-derived fluorophore concentration for phantoms A to I at $[\mathrm{PpIX}]=5 \mu \mathrm{g} / \mathrm{mL}$.

\begin{tabular}{lcc}
\hline & $\begin{array}{c}\text { rms Deviation } \\
\text { from Mean (\%) }\end{array}$ & $\begin{array}{c}\text { Maximum Deviation } \\
\text { from Mean (\%) }\end{array}$ \\
\hline $\begin{array}{l}\text { Fluorescence } \\
\text { image intensity }\end{array}$ & 56.5 & 150.2 \\
$\begin{array}{l}\text { Measured fluorescence } \\
F_{x, m}\left(\lambda_{m}=635 \mathrm{~nm}\right)\end{array}$ & 52.3 & 94.9 \\
Estimated $[\mathrm{PpIX}]$ & 10.1 & 14.2 \\
\hline
\end{tabular}

the tumor: bear in mind, however, that the tumors are only a few millimeters in diameter, so that there may be a contribution from the exact probe positioning.

\section{Discussion and Conclusions}

One of the main challenges of this work was to produce a simple analytic equation that models fluorescence measurements in tissue, and that can be rapidly computed to enable in situ application. The model presented here achieves this goal and, from initial clinical studies in progress, ${ }^{19}$ has been found to be highly practical in the real intraoperative environment. However, there are inherent limitations that are due to the assumptions made in the model.

Recapitulating, the assumptions used are (1) that reflectance and fluorescence photons traverse similar paths providing that the tissue absorption at the excitation wavelength is much larger than at the emission (detection) wavelengths (i.e., $\mu_{a, x} \gg \mu_{a, m}$ ), (2) that the intrinsic tissue absorption at the excitation wavelength is much larger than the added absorption due to the fluorophore (i.e., that $\mu_{a, x} \gg \mu_{a f, x}$ ), and (3) that hemoglobin is the dominant absorber in tissue. Assumptions 1 and 2 are used primarily in the quantitative fluorescence model [Eq. (5)] and were validated by the phantom experiments. Thus, applying the quantitative fluorescence model with known optical properties resulted in a significant ( $\sim$ fivefold) decrease in the rms deviation compared with the measured, uncorrected fluorescence signal for a given fluorophore concentration. The improvement in the coefficient of determination $\left(R^{2}=0.639\right.$ for the uncorrected data and 0.976 after applying the model) further supports the validity of the model, given accurate values of the tissue optical properties as inputs.

The ex vivo organ study was carried out mainly to test assumption 3 across different tissue types, i.e., to test whether the 
(a)

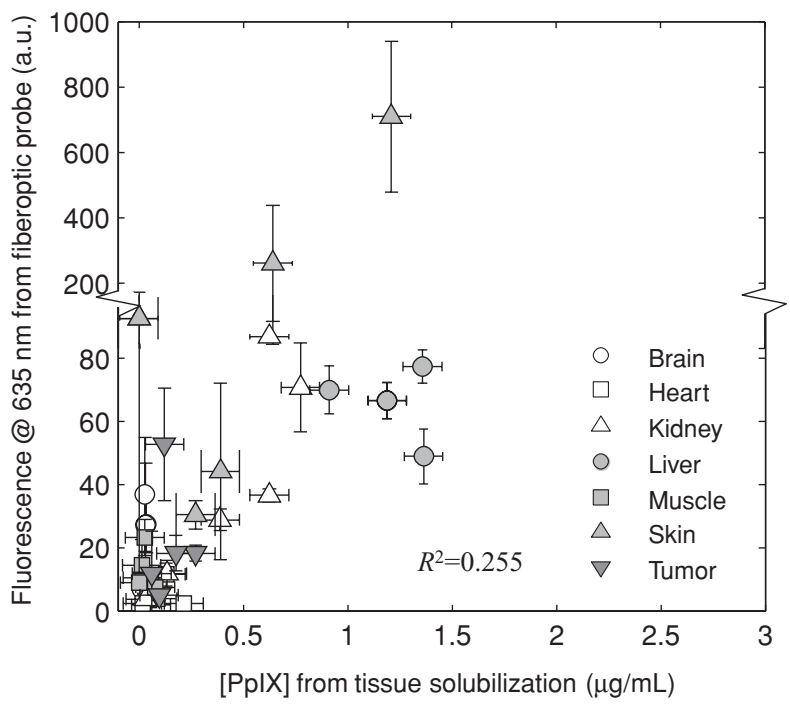

(b)

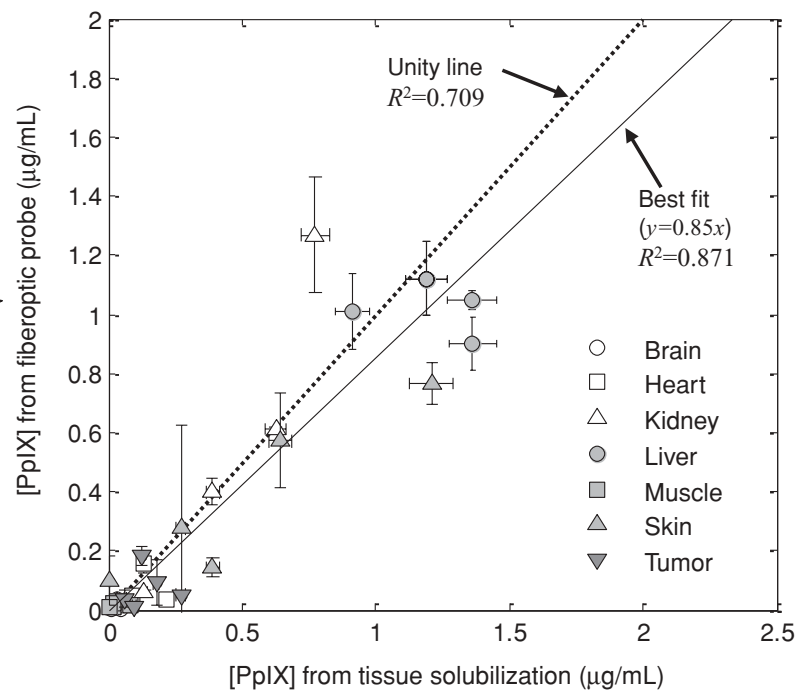

Fig. 7 Fluorescence measurements in ex vivo mouse tissues: (a) uncorrected fluorescence intensity at $635 \mathrm{~nm}$ versus PpIX concentration from the tissue solubilization data (the $y$ axis scale is in two parts to display better the range of data) and (b) model-derived [PpIX] values versus tissue solubilization values. The $Y$ axis error bars are \pm 1 standard deviation (s.d.) from three measurements on each tissue sample. The $X$ axis error bars were calculated from the rms errors estimated by Lilge et al. ${ }^{8}$

technique is "universal." From Fig. 7, the estimates of [PpIX] derived using the model indicate that absolute fluorophore measurements are feasible, notwithstanding residual errors for some tissue types. For example, liver and the B16 melanoma tumor are highly pigmented tissues, causing $\mu_{a}$ to be underestimated, which in turn leads to underestimation of the quantitative fluorescence, and indeed there seems to be a systematic bias for these two tissues as shown in Fig. 7(b). From Fig. 2(b), the factor $1 /\left(1-R_{t}\right)$ is nearly constant at low albedo (i.e., $\left.\alpha^{\prime}<0.5\right)$ so that, given that the reflectance spectrum at the emission wavelengths is measured correctly, the major factor that affects the fluorescence correction model [Eq. (5)] is the $\mu_{a, x}$ estimate. We surmise, therefore, that this technique is more suitable for tissues where hemoglobin dominates the absorption in the excitation band, such that $\mu_{a, x}$ is estimated accurately, rather than in highly pigmented tissues with additional chromophores. It may be possible to incorporate other chromophores into a more sophisticated spectrally constrained diffuse reflectance model by expanding Eq. (9). Another potential source of error in the

Table 3 PplX concentration measurements taken in vivo near the tumor injection site and in normal brain tissue.

\begin{tabular}{lcc}
\hline & $\begin{array}{c}\text { Near Tumor Cell Injection Site } \\
{[\mathrm{PpIX}] \text { in } \mu \mathrm{g} / \mathrm{mL}(\mathrm{max} / \mathrm{min}} \\
\text { for } N=10)\end{array}$ & $\begin{array}{c}\text { Contralateral Normal Brain } \\
{[\mathrm{PpIX}] \text { in } \mu \mathrm{g} / \mathrm{mL}(\mathrm{max} / \mathrm{min}} \\
\text { for } N=10)\end{array}$ \\
\hline Rabbit 1 & $1.081 / 0.004$ & $0.097 / 0.001$ \\
Rabbit 2 & $0.224 / 0.003$ & $0.013 / 0.001$ \\
Rabbit 3 & $1.951 / 0.017$ & $0.027 / 0.001$ \\
Rabbit 4 & $2.451 / 0.028$ & $0.010 / 0.001$ \\
\hline
\end{tabular}

murine tissue experiment is the heterogeneity in PpIX localization. Solubilizing the tissue has the effect of averaging this, whereas the fiber optic probe interrogates only a superficial region of the tissue of each organ.

Although the mouse organ experiment was not designed as a pharmacokinetics study, it is useful to compare the results with other data on ALA-PpIX, as summarized in Table 4. Thus, Sroka et al. injected mice with $50 \mathrm{mg} / \mathrm{kg}$ i.v. via the tail vein, ${ }^{20}$ while Moan et al. ${ }^{21}$ used intraperitoneal injection at $1.5 \mathrm{mM} / \mathrm{kg}$, and the results seem to be consistent (even though our data has only

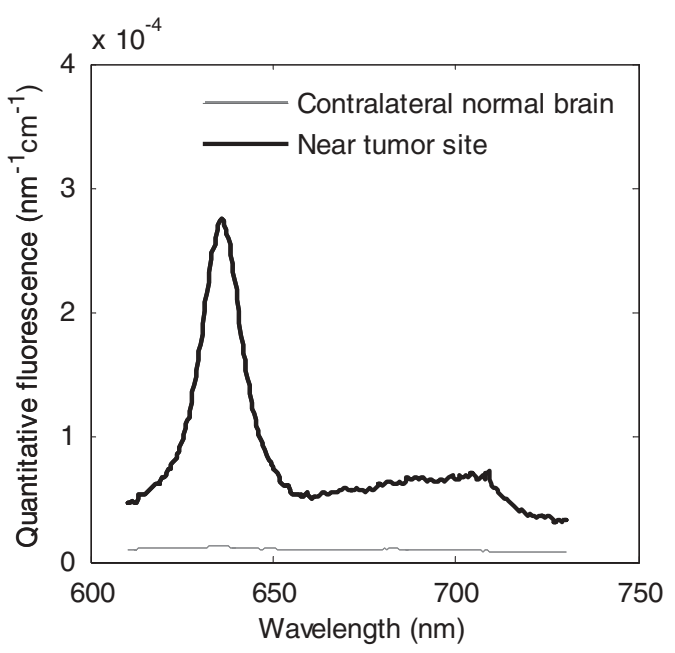

Fig. 8 Representative fluorescence spectra corrected for optical properties for one animal (rabbit 3), with the spectrum taken near the tumor injection site, showing enhanced PpIX levels compared with the spectrum taken from normal brain tissue; the corresponding model-derived PpIX concentrations were 1.951 and $0.016 \mu \mathrm{g} / \mathrm{mL}$ for the tumor and normal sites, respectively. 
Table 4 Comparison between PpIX kinetics measured in the murine tissue study in this work and other published data.

\begin{tabular}{|c|c|c|c|}
\hline \multirow[b]{2}{*}{ Tissue } & \multicolumn{3}{|c|}{ Time of max fluorescence $(h)$} \\
\hline & Our data & Sroka et al. ${ }^{20}$ & Moan et al. ${ }^{21}$ \\
\hline Brain & 1 & 0.9 & \\
\hline Kidney & 2 & 1 & \\
\hline Liver & 0.5 & 0.8 & \\
\hline Muscle & 0.5 & 0.8 & \\
\hline Skin & 3 & 3.1 & 4 \\
\hline
\end{tabular}

$N=1$ per time point). It is encouraging that there is no apparent significant systematic bias introduced by the fiber optic probe technique, so that it should be reliable for time-dependent or longitudinal studies. Note, however, that the measurements may be sensitive to pressure exerted by the probe tip on the tissue, since this can alter the local blood flow, so that gentle contact is required.

The rabbit tumor experiment also demonstrated that this technique works well in vivo for use in fluorescence-guided resection of brain tumors, our initial clinical application. Since the fluorescence signals had high SNRs (in the rabbit study the average SNR was $43 \mathrm{~dB}$ ) at this clinically relevant ALA dose ${ }^{2}$ $(20 \mathrm{mg} / \mathrm{kg})$, we believe that the technique will perform as expected in the clinical environment-indeed this is borne out by recent preliminary work. ${ }^{19}$ It is of interest to ask what fraction of tumor cell density in the tissue can be detected. This can be estimated from the rabbit brain tumor data in which the average tumor-to-normal contrast ratio was 35:1. If the detectability threshold is considered conservatively to be $5: 1$, then the technique should be able to detect $\sim 14 \%(5 \div 35 \times 100 \%)$ tumor "infiltrate."

In the ex vivo murine tissue and in vivo rabbit experiments, repeat measurements at the same tissue site were not made, so that the reproducibility of quantitative fluorescence measurements in tissues is not known. The quantitative fluorescence measurements may be affected, for example, by pressure differences of the probe on the tissue, as this may change the local blood content. However, to some extent the fluorescence measurement should be self-correcting, since the corresponding tissue attenuation used in Eq. (5) will change. As part of clinical trials in progress for glioma resection ${ }^{19}$ (with patients given ALA prior to surgery), multiple probe measurements are taken per tissue site to answer this critical question of measurement repeatability in vivo.

In conclusion, absolute and accurate quantification of fluorophore concentration in tissues in vivo is a challenging problem, complicated primarily by the (unknown) variations in tissue optical properties. In this work, we have focused on absolute quantification of the fluorescence spectrum to determine the true concentration of a target fluorescent species. The model is successful in reducing the distortion of the fluorescence spectrum and signal intensity due to tissue optical properties, when used under conditions of high tissue attenuation (380 to 450 $\mathrm{nm})$. We expect this method is capable, in principle, of quantifying many fluorophores other than PpIX, potentially including those comprising the tissue autofluorescence and those emitting in the shorter, 450 to $600-\mathrm{nm}$ wavelength range. In principle, the hemoglobin absorption bands in the 450 to 650 -nm range should not pose difficulty in the quantitative fluorescence model as long as the excitation wavelength is in the UV/blue region (i.e., that the inequality $\mu_{a, x}>\mu_{a, m}$ still holds); however, this must be experimentally verified. This technique is adept at measuring the concentration of PpIX in situ for a variety of murine tissues, as well as in the case of an in vivo tumor. This instrument is currently deployed in clinical trials in malignant glioma patients undergoing fluorescence-guided resection surgery mediated with ALA-PpIX.

\section{Acknowledgments}

The authors gratefully acknowledge the support from the National Institutes of Health, Grant No. R01NS052274-01A1, and support for A. Kim from the Natural Sciences and Engineering Research Council of Canada. Partial core support was provided by the Ontario Ministry of Health and Long-Term Care: the views expressed do not necessarily reflect those of the OMOHLTC.

\section{References}

1. J. C. Finlay, T. C. Zhu, A. Dimofte, D. Stripp, S. B. Malkowicz, T. M. Busch, and S. M. Hahn, "Interstitial fluorescence spectroscopy in the human prostate during Motexafin Lutetium-mediated photodynamic therapy," Photochem. Photobiol. 82, 1270-1278 (2006).

2. W. Stummer, U. Pichlmeier, T. Meinel, O. D. Wiestler, F. Zanella, H. J. Reulen, and the ALA-Glioma Study Group, "Fluorescence-guided surgery with 5 aminolevulinic acid for resection of malignant glioma: a randomized controlled multicentre phase III trial," Lancet Oncol. 7, 392-401 (2006).

3. M. G. Müller, I. Georgakoudi, Q. Zhang, J. Wu, and M. S. Feld, "Intrinsic fluorescence spectroscopy in turbid media: disentangling effects of scattering and absorption," Appl. Opt. 40, 4633-4646 (2001).

4. K. R. Diamond, M. S. Patterson, and T. J. Farrell, "Quantification of fluorophore concentration in tissue-simulating media by fluorescence measurements with a single optical fiber," Appl. Opt. 42, 2436-2442 (2003).

5. T. J. Pfefer, K. T. Schomacker, M. N. Ediger, and N. S. Nishioka, "Light propagation in tissue during fluorescence spectroscopy with single-fiber probes," IEEE J. Sel. Top. Quantum Electron. 7, 1004-1012 (2001).

6. J. Wu, M. S. Feld, and R. P. Rava, "Analytical model for extracting quantitative fluorescence in turbid media," Appl. Opt. 32, 3585-3595 (1993).

7. R. Weersink, M. S. Patterson, K. Diamond, S. Silver, and N. Padgett, "Noninvasive measurement of fluorophore concentration in turbid media with a simple fluorescence/reflectance ratio technique," Appl. Opt. 40, 6389-6395 (2001).

8. L. Lilge, C. O'Carroll, B. C. Wilson, "A solubilization technique for photosensitizer quantification in ex vivo tissue samples," J. Photochem. Photobiol. B 39(3), 229-235 (1997).

9. A. Kim, M. Roy, F. Dadani, and B. C. Wilson, "A fiberoptic reflectance probe with multiple source-collector separations to increase the dynamic range of derived tissue optical absorption and scattering coefficients," Opt. Express 18, 5580-5594 (2010).

10. W. Stummer, A. Novotny, H. Stepp, C. Goetz, K. Bise, and H. J. Reulen, "Fluorescence-guided resection of glioblastoma multiforme by using 5-aminolevulinic acid-induced porphyrins: a prospective study in 52 consecutive patients," J. Neurosurg. 93, 1003-1013 (2000).

11. M. S. Eljamel, C. Goodman, and H. Moseley, "ALA and Photofrin fluorescence-guided resection and repetitive PDT in glioblastoma 
Kim et al.: Quantification of in vivo fluorescence decoupled from the effects...

multiforme: a single centre phase III randomised controlled trial," Lasers Med. Sci. 23, 361-367 (2008).

12. V. X. Yang, P. J. Muller, P. Herman, and B. C. Wilson, "A multispectral fluorescence imaging system: design and initial clinical tests in intra-operative Photofrin-photodynamic therapy of brain tumors," Lasers Surg. Med. 32, 224-232 (2003).

13. S. T. Flock, M. S. Patterson, B. C. Wilson, and D. R. Wyman, "MonteCarlo modeling of light-propagation in highly scattering tissues: 1. Model predictions and comparison with diffusion-theory," IEEE Trans. Biomed. Eng. 36, 1162-1168 (1989).

14. F. A. J. Groenhuis, H. A. Ferwerda, and J. J. ten Bosch, "Scattering and absorption of turbid materials derived from reflection coefficients. 1: Theory," Appl. Opt. 22, 2456-2462 (1983).

15. A. Kim and B. C. Wilson, "Measurement of ex vivo and in vivo tissue optical properties: methods and theories," Chap. 8 in Optical-Thermal Response of Laser-Irradiated Tissue, A. J. Welch and M. J. C. van Gemert, Eds., Dordrecht: Springer SBM (2010).

16. S. L. Jacques, "Light distributions from point, line, and plane sources for photochemical reactions and fluorescence in turbid biological tissues," Photochem. Photobiol. 67, 23-32 (1998).
17. D. S. Kepshire, S. L. Gibbs-Strauss, J. A. O'Hara, M. Hutchins, N. Mincu, F. Leblond, M. Khayat, H. Dehghani, S. Srinivasan, and B. W. Pogue, "Imaging of glioma tumor with endogenous fluorescence tomography," J. Biomed. Opt. 14, 030501 (2009).

18. L. Lilge and B. C. Wilson, "Photodynamic therapy of intracranial tissues: a preclinical comparative study of four different photosensitizers," J. Clin. Laser Med. Surg. 16, 81-91 (1998).

19. P. A. Valdes, F. Leblond, A. Kim, B. T. Harris, B. C. Wilson, X. Fan, T. D. Tosteson, A. Hartov, S. Ji, K. D. Paulsen, and D. W. Roberts, "Quantitative fluorescence in intracranial tumor: implications for ALAinduced PpIX as an intraoperative biomarker," J Neurosurg (in press, 2010).

20. R. Sroka, W. Beyer, L. Gossner, T. Sassy, S. Stocker, and R. Baumgartner, "Pharmacokinetics of 5-aminolevulinic-acid-induced porphyrins in tumour-bearing mice," J. Photochem. Photobiol. B Biol. 34, 13-19 (1996).

21. J. Moan, L.-W. Ma, A. Juzeniene, V. Iani, P. Juzenas, F. Apricena, and Q. Peng, "Pharmacology of protoporphyrin IX in nude mice after application of ALA and ALA esters," Intl. J. Cancer 103, 132-135 (2003). 